\title{
Reflexión sobre la precariedad laboral y sus consecuencias desde un enfoque de género
}

\author{
Esperanza Vargas Jiménez ${ }^{1}$ \& Claudia Gregoria Huerta Zúñiga ${ }^{2}$
}

Recibido:11/10/2018 Aceptado: 26/08/2019

DOI: $10.21772 /$ ripo.v38n2a01

\begin{abstract}
Resumen
Diversos organismos nacionales e internacionales, sindicatos, la comunidad académica y sociedad en general han denunciado el deterioro de las condiciones laborales, principalmente se critica que para maximizar las ganancias de los empleadores se han menoscabado algunos derechos laborales a través de bajos salarios, menor acceso a seguridad social, el aumento de contratos temporales en detrimento de contratos definitivos de trabajo y escasa certidumbre acerca de la continuidad del trabajo. En el presente documento se analiza el concepto de trabajo precario, posteriormente se describen las múltiples dimensiones o manifestaciones de este fenómeno y se aborda la importancia de estudiar esta temática; posteriormente se exponen algunas de las principales consecuencias de la precariedad para quienes lo viven, después se indaga cómo la precariedad afecta de forma distinta a hombres y mujeres, después se exploran algunas teorías que explican la diferenciación por género, por último, se despliegan las conclusiones del análisis realizado.
\end{abstract}

Palabras clave: Precariedad laboral, consecuencias psicosociales, género.

\section{Reflection on Labor Precariousness and its Consequences from a Gender Perspective}

\begin{abstract}
Various national and international organizations, unions, the academic community and society in general have denounced the deterioration of working conditions, it's mainly criticized that to maximize the profits of employers some labor rights have been undermined through low wages, lesser access to social security, the increase of temporary contracts to the detriment of definitive work contracts and meager certainty about the continuity of work. In this document, the concept of precarious work is analyzed, later the multiple dimensions or manifestations of this phenomenon are described and the importance of studying this topic is discussed, subsequent some of the main consequences of precariousness for those who live it are exposed, afterwards, it is investigated how precariousness affects men and women differently, then, some theories that explain this gender differentiation are explored, finally, the conclusions of the analysis are displayed.
\end{abstract}

Key words: Job precariousness, psychosocial consequences, gender

\footnotetext{
1 Dra. en Ciencias para el Desarrollo Sustentable. Universidad de Guadalajara. Profesor docente investigador titular A. E-mail: esperanzavgas@ hotmail.com

2 Ms. en Ciencias para el Desarrollo, la Sustentabilidad y Turismo. Universidad de Guadalajara. Estudiante del doctorado en Ciencias para el desarrollo, la sustentabilidad y el turismo. E-mail: claughz@hotmail.com

Cómo citar este artículo: Vargas Jiménez, E. \& Huerta Zúñiga, C. G. (2019). Reflexión sobre la precariedad laboral y sus consecuencias desde un enfoque de género. Revista Interamericana de Psicología Ocupacional, 38(2), 104-114. DOI: 10.21772/ripo.v38n2a01
} 


\section{Introducción}

El trabajo es un aspecto importante de la vida adulta por ser una forma de sustentarse y encontrar sentido de vida, Bauman (1999) advierte que el consumismo y la globalización ha transformado la forma en que se concibe el trabajo, señala que anteriormente era concebido como una expresión individual y actualmente es un medio para poder consumir todo lo que el mercado y la élite del capitalismo nos convence que necesitamos para lograr vivir bien, el autor explica que esta transición del trabajo está directamente relacionado con el esfuerzo por aumentar la producción y las ganancias; en este sentido, Bauman subraya que también se ha transformado la concepción de la pobreza, en tiempos pasados ser pobre era sinónimo de no tener trabajo y hoy en día ser pobre es no tener la posibilidad de ser consumidor potencial, es decir, estar expulsado del mercado.

Con relación al caso específico de América Latina Castel (2000) sostiene que el gran problema es el fenómeno del subempleo, el cual define como un cuello de botella caracterizado por malas condiciones de trabajo, de salario y de explotación, en la misma línea. Ahora bien, el autor denuncia que se ha producido un cambio decisivo relacionado con la flexibilización del empleo y la finalización del empleo estable y de larga duración con la desaparición de contratos indefinidos que son sustituidos por contratos temporales.

La Organización Internacional del Trabajo (OIT, 2012) advierte de los numerosos riesgos de un fenómeno conocido como precariedad laboral. Postula que se puede manifestar de diversas formas y advierte de la necesidad de erradicar la precariedad como requisito indispensable para mejorar la calidad de vida de los trabajadores y para alcanzar tanto el crecimiento económico como el desarrollo de los países. En primer lugar, es importante tener claridad de qué se entiende por precariedad laboral. La OIT al definir el trabajo precario plantea que:

...es un medio utilizado por los empleadores para trasladar los riesgos y las responsabilidades a los trabajadores. Es el trabajo que se realiza en la economía formal e informal y que se caracteriza por niveles variables y grados de particularidades objetivas (situación legal) y subjetivas (sensación) de incertidumbre e inseguridad. [...] se lo suele definir por la incertidumbre que acarrea en cuanto a la duración del empleo, la presencia de varios posibles empleadores, una relación de trabajo encubierta o ambigua, la imposibilidad de gozar de la protección social y los beneficios que por lo general se asocian con el empleo, un salario bajo y obstáculos considerables tanto legales como prácticos para afiliarse a un sindicato y negociar colectivamente (OIT, 2012, p. 32).

De esta definición podemos destacar algunos puntos clave, en primer lugar, señala que los empleadores evaden su responsabilidad, es decir, buscan lagunas legales que les permiten tener las menores obligaciones posibles con sus trabajadores, traduciéndose esto en menor pago de impuestos y de prestaciones, por lo tanto, en mayores ganancias para los empleadores. De la propia definición se infiere la responsabilidad de las autoridades gubernamentales para erradicar este fenómeno de raíz legislando políticas públicas y normas legales que protejan los derechos laborales y vigilando que dichas normas sean cumplidas a cabalidad por las empresas; además, hay una responsabilidad importante de los sindicatos de trabajadores por velar que efectivamente se cumpla la legislación ya existente al respecto y denunciar las irregularidades en los contratos colectivos y/o individuales de trabajo, esto con el objetivo de que no se continúe vulnerando los derechos de los trabajadores. 
Aunado a ello, la definición anteriormente expuesta señala que el trabajo precario se caracteriza tanto por ideas objetivas relacionadas con el entorno o contexto (por ejemplo, la situación legal del trabajador con la empresa) como subjetivas, que se refieren a la percepción del trabajador y sus experiencias con respecto a su trabajo. Esta conceptualización distingue entre las ideas y pensamientos con respecto a la pérdida de trabajo, por un lado, y los sentimientos y miedos asociados con esa cognición, por el otro (Pienaar, De Witte, Hellgren \& Sverke, 2013); es precisamente la parte subjetiva del fenómeno de la precariedad la que es más compleja de analizar y se refiere a la incertidumbre acerca del futuro del empleo o la percepción de desprotección.

Por último, esta definición de la OIT manifiesta que la precariedad laboral tiene diversas consecuencias para el trabajador que afectan su bienestar y su calidad de vida, por ejemplo, bajos salarios, no contar con protección social e inclusive la imposibilidad de afiliarse a un sindicato que luche por la mejora de sus condiciones laborales y por supuesto incertidumbre acerca del futuro de su trabajo que puede derivar en ansiedad, depresión, estrés y malestar; esta es la parte de la precariedad laboral que reviste de gran interés para la psicología.

Debido a la forma en que este fenómeno trastoca la calidad de vida de las personas, la erradicación de la precariedad laboral se ha vuelto indispensable para alcanzar el desarrollo, por ello, la Organización de las Naciones Unidas (ONU) incorpora en el objetivo número 8 de los Objetivos del Desarrollo Sostenible (ODS) la búsqueda del "trabajo decente", en dicho objetivo se exhorta a los países miembros de la ONU a crear las condiciones adecuadas para que la población pueda acceder a empleos de calidad que garanticen niveles adecuados de calidad de vida para los trabajadores, no solo se refiere a empleos bien remunerados, sino empleos que generen bienestar.

\section{Desarrollo del tema}

\section{Dimensiones de la precariedad}

Existen diversas manifestaciones de la precariedad laboral, tales como el empleo informal, el trabajo sin contrato, largas jornadas de trabajo, contratos temporales, utilización de empresas de outsourcing, entre otras formas de flexibilidad laboral (Neffa, 2010). La OIT postula que los tipos de precariedad varían de acuerdo con el contexto específico de cada país, aunado a ello advierte que continúan expandiéndose y surgiendo nuevas formas de precariedad laboral debido a que los empleadores siguen buscando y descubriendo maneras para evadir regulaciones existentes o encuentran vacíos legales que le permiten incrementar sus ganancias o rentabilidad a costa de sus empleados (OIT, 2012). De esta afirmación se infiere que las políticas públicas relacionadas con el empleo deben tener una regulación mucho más estricta para evitar que algunos empleadores evadan la ley y trastoquen los derechos laborales en su afán de obtener mayores ganancias, además el combate a la corrupción y a la impunidad se vuelve imprescindible para evitar que más tipos de precariedad laboral se sigan expandiendo.

La literatura científica actual reconoce cuatro principales dimensiones de precariedad, para explicarlas detalladamente se seguirán los argumentos de Gutiérrez (2016), quien al desglosar dichas dimensiones explica que la primera corresponde a la inseguridad sobre la continuidad de la relación laboral. Esta primera característica de la precariedad se refiere a la incertidumbre sobre el futuro del trabajo, que puede o no estar fundada en aspectos objetivos como el tipo de contrato o una situación específica dentro del lugar de trabajo. Para ejemplificar este tipo de empleos podemos pensar en trabajos temporales, clandestinos o bien empleos que tienen un alto riesgo de desaparecer. Es decir, son empleos cuya característica principal es la ausencia de certeza sobre la continuidad de este, en cualquier momento el empleador puede prescindir de los 
servicios de su empleado sin serias repercusiones legales o económicas debido a la ausencia de un contrato definitivo de trabajo que le permita al trabajador acceder a una liquidación adecuada.

Al abordar las consecuencias de esta característica de la precariedad Gutiérrez (2016) manifiesta que la inseguridad sobre la continuidad obstaculiza que el trabajador tenga control de su futuro laboral, poniéndolo en una posición vulnerable en su relación con la empresa y su empleador. Lo que implica que el trabajador pierde poder y control sobre su propio trabajo y es el empleador quién ejerce dicho poder y tiene la capacidad de decidir arbitrariamente sobre el futuro del trabajo sin mediación de sindicatos $\mathrm{u}$ otros organismos. Una de las características principales de un trabajo con buenas condiciones laborales tiene que ver con que el trabajador tenga asegurada la continuidad de su empleo, por lo tanto, se relaciona directamente con el tipo de contrato que tenga el trabajador, esto debido a que si cuenta con un contrato temporal el empleado no tiene forma de conocer si una vez terminado el contrato podrá continuar con su trabajo, exponiéndolo así en una situación de precariedad.

La segunda dimensión de la precariedad laboral se relaciona con la insuficiencia de los ingresos salariales, es decir, bajo salario. La remuneración económica tiene un gran impacto porque se relaciona íntimamente con el nivel de vida, la autonomía, el control sobre el futuro y hasta la posición social (Agulló-Tomás, 1998; Gutiérrez, 2016). Por último, cabe señalar que esta insuficiencia salarial es una forma de perpetuación de la pobreza y dificulta la conciliación familiar, ya que si el salario de un solo trabajo no alcanza para cubrir las necesidades básicas es probable que se busque un segundo empleo disminuyendo el tiempo de ocio, descanso y de convivencia familiar.

La tercera dimensión está vinculada con las condiciones específicas del trabajo (jornada laboral, ritmo de trabajo, salud, posibilidades de promoción y riesgos de trabajo), incluye diversas formas en las que se puede vulnerar las condiciones laborales, cabe mencionar que estas formas de degradar los derechos laborales se relacionan íntimamente con la capacidad de los empleadores para evadir las regulaciones legales que rigen las relaciones laborales.

La cuarta y última dimensión es la reducción de la protección social para el trabajador, esta dimensión explica el por qué se facilita a los empleadores evadir la ley y no responsabilizarse con sus trabajadores, la ley se vuelve flexible y amigable para las empresas, aumentando la precariedad laboral y facilitando que evasión de la responsabilidad a través de diversas lagunas legales. Es importante destacar que en esta dimensión resalta la responsabilidad de las autoridades gubernamentales al no garantizar la protección social mediante dos métodos: pobre regulación legal con respecto a las relaciones laborales y falta de cobertura de los servicios de protección legal.

\section{La importancia de analizar el trabajo precario}

Al analizar el mercado laboral surgen diversas temáticas que serían indispensables abordar y estudiar como la erradicación del desempleo, sin embargo, para mejorar las condiciones laborales no se requiere únicamente crear más empleos, también es importante saber qué calidad tienen los empleos ya existentes; al respecto, y explicando la importancia de analizar el trabajo precario, la OIT $(2012$, p. 3) manifiesta que:

Las cifras de desempleo provocan preocupación por sí mismas, pero ni siquiera logran abarcar a una mayoría más amplia de personas que trabajan pero que no tienen un empleo decente con salarios dignos, futuro estable, protección social y acceso a derechos fundamentales. Es innegable que la universalidad $y$ las 
dimensiones del conflicto exigen una acción coordinada y abarcadora a nivel internacional.

Es decir, en términos estadísticos de frecuencia, existen más personas con trabajo precario que sin trabajo, esto quiere decir que aun cuando tienen empleo éste no les proporciona un bienestar ni les garantiza las necesidades más básicas, mucho menos les asegura una buena calidad de vida. Esto es preocupante, pareciera que los derechos de los trabajadores han perdido por completo su importancia y son sacrificados en aras de optimizar las ganancias. Además, la OIT (2012) hace énfasis en el carácter universal de este tipo de empleos, es decir, la precariedad no es exclusiva de ciertas zonas o de ciertos países, sino que es un fenómeno extendido en todo el mundo.

\section{Consecuencias de la precariedad laboral}

Con relación a las consecuencias de la precariedad laboral, Canivet et al. (2017, p. 517) revelan que:

Las investigaciones y el interés por estudiar la salud mental y el trabajo precario han aumentado rápidamente durante la década pasada, recientes análisis han probado una relación entre problemas de salud mental, trabajo precario y trabajo temporal. Los efectos del trabajo precario difieren significativamente dependiendo de la edad, la exposición a la situación del mercado laboral tiene un significado diferente dependiendo de la etapa de la carrera profesional. La precariedad laboral tiene efectos más débiles entre los jóvenes que en otros grupos de edad.

Una de las principales consecuencias de la precariedad laboral es una disminución de la salud mental, se pudiera pensar que las consecuencias de las malas condiciones del trabajo son meramente económicas, sin embargo, es importante para futuras investigaciones preguntarse si los trabajadores que experimentan incertidumbre laboral tienen niveles más altos de ansiedad, estrés, o alteraciones en la salud mental, que pudieran disminuir su productividad y calidad de vida.

Abonando al entendimiento de la relación entre la salud mental y el trabajo precario Moscone, Tosetti, Vittadini (2016) revelan que, de acuerdo con los datos encontrados en su investigación la probabilidad ser prescrito con medicamento psiquiátrico aumenta para aquellos trabajadores con contratos de trabajo temporal. Esta afirmación revela la importancia de las condiciones laborales en la salud física y mental de las personas, el trabajo no es únicamente una forma de sustento de las personas, también nos da sentido de vida, una identidad y sentido de pertenencia, formando así parte importante del desarrollo social de las personas y de la sociedad (Agulló-Tomás, Llosa, \& Agulló-Tomás, 2018; Blanco, 1997), por lo tanto, cuando las condiciones laborales son precarias se afectan diferentes aspectos y principalmente la salud mental.

Continuando con la explicación de por qué la precariedad afecta la salud mental, Moscone, Tosetti, Vittadini (2016, p. 86) manifiestan que:

Más días de trabajo en los contratos temporales, así como los frecuentes cambios en los contratos temporales aumentan significativamente la probabilidad de desarrollar problemas de salud mental que requieran ser tratados medicamente. También encontramos que pasar de trabajo permanente a trabajo temporal aumenta las enfermedades mentales; simétricamente, aunque con un menor efecto, pasar de trabajo temporal a trabajo permanente tiende a reducir las enfermedades mentales.

Esto implica que el tipo de contrato, factor importante para evaluar la precariedad 
laboral, también influye directamente en la salud mental. Un empleo inestable y temporal conlleva incertidumbre $\mathrm{y}$ dicha incertidumbre puede afectar significativamente la vida de las personas de tal forma que su salud mental se vea deteriorada e incluso pueda requerir medicamento psiquiátrico. Llama especialmente la atención que pasar de trabajo temporal a trabajo permanente aumente la salud mental, comprobándose que la incertidumbre respecto a la continuidad del trabajo es un factor que deteriora la salud mental, y se infiere que es precisamente esta parte subjetiva de la precariedad la que tiene mayor impacto en el bienestar de las personas.

\section{Diferencias entre hombres y mujeres en la precariedad laboral}

La OIT (2012) reconoce que hay grupos más propensos a sufrir precariedad laboral, entre los cuales se encuentran los jóvenes, las mujeres y las personas con discapacidad; en la presente reflexión el foco se centra en las diferencias existentes entre hombres y mujeres al experimentar la precariedad laboral, esto debido a la imposibilidad de abordar todos los aspectos de la precariedad y por considerar que el enfoque de género debe ser un eje transversal al momento de analizar los diversos fenómenos sociales. En su investigación, Young (2010, p. 91) explica que:

La sobrerrepresentación de las mujeres en el trabajo precario también es una función de las prácticas discriminatorias del empleador [...] En comparación con las mujeres, es menos probable que los hombres trabajen a tiempo parcial. Y, en comparación con los hombres, las mujeres que pasan el mismo tiempo en las tareas del hogar tienen más probabilidades de ser penalizadas, reciben menos salario y tienen más probabilidades de trabajar a tiempo parcial. Estos hallazgos sugieren que es más probable que las mujeres sean empleadas en trabajos precarios como resultado de las preferencias discriminatorias de los empleadores.

Lo anterior implica que, a pesar del carácter universal de la precariedad, las mujeres continúan viviendo desigualdad en el contexto laboral y mayormente parciales, el autor hace una breve explicación con relación a por qué las mujeres son más propensas a la precariedad, sin embargo, esta hipótesis no considera la responsabilidad de las autoridades de diversos órdenes de gobierno que no han fomentado políticas públicas que prevengan este tipo de discriminación en el trabajo, pareciera que se responsabiliza en mayor medida al empleador y se deja de lado las condiciones del contexto que facilitan que exista dicha discriminación, incluyendo la poca regulación que existe que permite que dichos empleadores trasgredan los derechos de sus trabajadores.

En muchas ocasiones se da por hecho que solo con que las mujeres se incorporen al mercado laboral es suficiente, las evidencias nos han probado que también se trata de mejorar las condiciones en las que trabajan; resalta especialmente el hecho de que las mujeres continúen siendo penalizadas por cuestiones relacionadas con su vida familiar, por ejemplo, la maternidad.

En su "informe mundial sobre salarios 2018/2019" la OIT (2019) expone, a partir de datos de 73 países, que la brecha salarial de género (ponderada) a nivel mundial es del alrededor del $16 \%$.

Un informe de la Organización de las Naciones Unidas (ONU, 2015) corrobora la vulnerabilidad de las mujeres en el mercado laboral, el organismo subraya que las mujeres perciben en promedio $24 \%$ menos de salario que los hombres, además, el $85 \%$ de los 92 países que mantienen registro con relación a las tasas de desempleo coinciden en que las mujeres con un elevado nivel académico tienen tasas de desempleo más altas que los hombres con el mismo 
nivel educativo. Lo anterior implica una merma importante en las oportunidades profesionales de las mujeres, quienes tienen menor oportunidad de acceder a puestos de alta cualificación, pese a su preparación académica y a que la tasa de incorporación al mercado laboral se acerca cada vez más a la de los hombres.

Percibir menor salario que los hombres, aún con la misma preparación y realizando las mismas labores, es una de las múltiples formas de exclusión y discriminación hacia las mujeres en el ámbito laboral, las estadísticas indican que, en los países miembros de la OCDE, las mujeres ganan en promedio casi un $15 \%$ menos que los varones (OCDE, 2017b), aún con el mismo puesto y mismo nivel educativo.

Con respecto al tipo de trabajo que las mujeres obtienen, la OCDE (2017a) indica que las mujeres tienen menores probabilidades de ser empresarios, además, aquellas empresas lidereadas por mujeres tienden a ganar menos que las que son propiedad de hombres, es decir, son mayoritariamente empleadas y aún aquellas autoempleadas se enfrentan a mayores condiciones adversas que los hombres autoempleados.

Para complementar esta información, la OCDE ofrece cifras del 2016 en las que se informa que en México el porcentaje de mujeres que son gerentes es de $34.3 \%$ y la diferencia salarial entre hombres y mujeres es de 16.7\% (OCDE, 2017a), lo que permite tener una idea de a qué tipo de trabajo son a los que acceden las mujeres, es decir, se ocupan en puestos mal remunerados y de baja cualificación.

Es importante señalar que, de acuerdo con la Encuesta Nacional de Ocupación y Empleo (INEGI, 2016), en el cuarto trimestre de 2015, la tasa de participación económica de las mujeres de 15 años y más con al menos un hijo fue de $44.2 \%$ y $96.7 \%$ y éstas combinan sus actividades empleo con los quehaceres domésticos.

En México, el Consejo Nacional para la
Evaluación de la Política de Desarrollo Social (CONEVAL, 2018) reconoce al trabajo doméstico no remunerado y de cuidado como uno de los factores que acentúan la desigualdad en las condiciones de vida de hombres y mujeres, siendo las mujeres quienes asumen esta sobrecarga de trabajo, para sustentar lo anterior el organismo informa que, en promedio, las mujeres dedican al trabajo doméstico o quehaceres del hogar entre 12 y 17 horas semanales más que los hombres, y entre 5 y 14 horas semanales más al cuidado exclusivo y sin remuneración de otras personas, como menores, adultos mayores o enfermos, dentro o fuera del hogar.

Abonando a las cifras estadísticas oficiales, Young (2010) afirma que son las mujeres quienes se dedican en mayor medida a las labores del hogar, esto es un factor importante al momento de ahondar acerca de la precariedad laboral debido a que el cuidado doméstico es un trabajo no remunerado, poco reconocido, desgastante físicamente y que se atribuye principalmente a las mujeres. El hecho de que sean las mujeres quienes se dediquen en mayor medida a las tareas del hogar pone en evidencia la existencia de estereotipos que perpetúan la idea de ocupaciones feminizadas y masculinizadas, es decir, de segregación ocupacional.

Siguiendo esta misma línea, Gobillon, Meurs \& Roux (2015), encontraron en su investigación que hay muy pocas mujeres en aquellos puestos bien remunerados, es decir, se encuentran subrepresentadas en las ocupaciones de más alta jerarquía, añaden que este hecho puede clarificar el por qué ha persistido la brecha salarial entre hombres y mujeres. Es decir, los datos científicos y confiables con los que contamos comprueban que efectivamente las mujeres tienen menor acceso a trabajos bien remunerados, haciéndolas más propensas a laborar en empleos precarios, de baja cualificación y con bajos salarios, hecho que pudiera explicar el por qué en promedio las mujeres ganan menos que los hombres, y pone en evidencia que persiste la 
inequidad de género en el mercado laboral.

\section{Teorías explicativas de las diferencias por sexo en la precariedad laboral}

Existen dos teorías principales que explican por qué las mujeres tienen mayor tendencia a la precariedad laboral, la primera es la teoría del capital humano que postula que las mujeres se comprometen en mayor medida con sus compromisos familiares actuales o esperados, por lo tanto, invierten menos tiempo en sus carreras profesionales, menguando su experiencia laboral, capacitación y resultados (Young, 2010). Es decir, esta primera teoría nos dice que las mujeres invierten menos tiempo, dinero y preparación a su profesionalización y capacitación e invierten en mayor medida en su familia, por lo tanto, aumentan la probabilidad de trabajar en entornos precarios.

La segunda propuesta para explicar por qué las mujeres tienen más trabajo precario, es la teoría de estratificación de género, esta teoría sugiere que las preferencias discriminatorias de los empleadores (hipotetizamos que estas preferencias pueden estar influidas por los estereotipos de género) influyen en la decisión al momento de elegir a quiénes contrata, por lo tanto, las mujeres son relegadas a puestos de baja cualificación, menor salario y a trabajos de tiempo parcial (Young, 2010), lo que se conoce como los sticky floors o pisos pegajosos que mantienen a las mujeres en la base de la pirámide económica. Así mismo, este postulado denuncia que las mujeres son castigadas por el tiempo que le dedican a su familia, es decir, se les niegan oportunidades laborales para acceder a mejores condiciones laborales debido a sus compromisos familiares. Esta teoría explicaría también la prevalencia de mujeres en puestos que reproducen tareas domésticas y de cuidado, por ejemplo: lavandería, camaristas, enfermeras, lavaplatos o cocineras (Díaz-Carrión, 2013; Lara-Aldave \&
Vizcarra-Bordi, 2008; Martínez, 2003; Mendoza \& Chapulín, 2015; Soares et al., 2005; Vargas, 2010).

Aportando al entendimiento de las razones que llevan a las mujeres a ocupar en mayor medida trabajos precarios, Gobillon, Meurs \& Roux (2015, p. 350) exponen tres ideas principales:

En primer lugar, las mujeres probablemente solicitan en menor medida para trabajos bien remunerados porque las jornadas laborales de este tipo de trabajo son menos compatibles con la vida familiar. En segundo lugar, puede haber discriminación sutil contra las mujeres que aumenta con el rango. En tercer lugar, puede haber una discriminación estadística tal que la distribución de habilidades sea la misma para hombres y mujeres, pero las habilidades son observadas con mayor incertidumbre o desconfianza para las mujeres que para los hombres por parte de los gerentes. De hecho, los hombres altamente calificados son contratados más frecuentemente que las mujeres altamente calificadas para trabajos bien remunerados.

Destaca que los autores no descartan que sean las propias mujeres quienes eligen no obtener puestos mejor remunerados (y posiblemente de mayor rango) debido a las responsabilidades familiares, argumento que coincide con el hecho de que sean las mujeres quienes se ocupan en mayor medida de las labores del hogar y de la crianza de los hijos, llevándolas a elegir sacrificar su vida profesional para atender su vida familiar, algo que no sucede en las decisiones de la mayoría los hombres. Es importante mencionar que esta explicación concuerda con la teoría de estratificación de género al resaltar que los empleadores perciben en menor medida las habilidades de las mujeres, es decir, científicamente no hay nada que pruebe que las mujeres tengan habilidades inferiores o superiores a la de los hombres, sin embargo, se 
reconocen en mayor medida las habilidades para ciertos puestos de trabajo en los hombres que en las mujeres.

\section{Conclusiones}

El trabajo precario es un fenómeno actual cuya característica principal es la incertidumbre con respecto a la duración del empleo, bajos salarios y desprotección legal; la incertidumbre que acarrea la precariedad se debe principalmente a una relación de trabajo ambigua y tiene diversas consecuencias, especialmente relacionadas con la dificultad para conciliar la vida familiar con la vida laboral por las largas jornadas, limitación para cubrir con todas las necesidades básicas debido al bajo salario y también consecuencias psicológicas que incluyen un deterioro significativo en la salud mental.

El desarrollo de los países no se puede lograr plenamente mientras siga existiendo la precariedad laboral por qué afecta el bienestar y la calidad de vida de las personas, además implica la perpetuación de la pobreza al ofrecer bajos salarios y limitar la posibilidad de ascenso profesional. El empleo no es únicamente un medio de sustento, es también una forma de obtener identidad y sentido de vida (Agulló-Tomás, Llosa, \& Agulló-Tomás, 2018; Blanco, 1997), consecuentemente, un trabajo con mejores condiciones laborales brinda bienestar y no únicamente sustento, mejorando así la salud mental.

Aunado a la importancia de afrontar la precariedad laboral nos encontramos con un fenómeno adicional: las mujeres son afectadas de forma particular por la precariedad. Se ha probado a través de las estadísticas oficiales y por medio de estudios científicos que las mujeres tienden en mayor medida que los hombres a encontrarse en empleos de baja cualificación, mal remunerados y de largas jornadas.

También existe otro fenómeno relacionado con la precariedad laboral, debido a que son las mujeres quienes en mayor medida se ocupan de las tareas domésticas y de cuidado, son ellas quienes tienen mayores dificultades con relación a la conciliación entre familia y trabajo. Autores como Gobillon, Meurs \& Roux (2015) señalan que inclusive es posible que las mujeres acepten voluntariamente trabajos precarios si éstos les permiten balancear de mejor manera la vida familiar y el trabajo, lo que merma su crecimiento profesional, perpetua la pobreza y coloca a este colectivo en una posición vulnerable.

Es importante ahondar en el hecho de que las mujeres sean mayormente afectadas por la precariedad por qué para alcanzar plenamente la equidad de género no es suficiente con que las mujeres accedan al mercado laboral, también es indispensable que tengan las mismas oportunidades en este ámbito que los hombres. Existen explicaciones interesantes que intentan comprender la razón de que sean las mujeres las mayormente afectadas por el trabajo precario, llama especialmente la atención cómo estás teorías relacionan íntimamente la precariedad laboral con la vida familiar, es decir, se postula que la necesidad de conciliar la vida familiar (en especial la crianza de los hijos y las tareas del hogar) se relaciona con el hecho de tener menores oportunidades en el ámbito laboral, debido a que son las mujeres quienes se ocupan en mayor medida de las tareas del hogar y de la crianza esta dificultad para conciliar ambos ámbitos afecta en mayor medida a este grupo, dando como resultado peores condiciones laborales para ellas.

Otra explicación evidencia una discriminación hacia las mujeres en el mercado laboral, esta teoría manifiesta que los empleadores no ven de igual manera las habilidades de los hombres que de las mujeres (fenómeno que se conoce como "techos de cristal"), por lo tanto, le ofrecen los empleos de mayor rango o jerarquía a los hombres, relegando los puestos de menor rango, y por lo tanto peor pagados, a las mujeres. 
La precariedad laboral es un fenómeno muy complejo que puede ser estudiado desde diversas aristas, sin embargo, no se debe perder de vista que afecta de manera particular a un colectivo, las mujeres, tratar de comprender la precariedad sin considerar este aspecto sería un enfoque reduccionista que impediría el avance en la lucha por el trabajo decente, algo fundamental para que los países alcancen el desarrollo pleno.

Futuras líneas de reflexión e investigación podrían orientarse hacía lo que sucede con otros grupos vulnerables como personas con discapacidad o jóvenes, o bien analizar concretamente lo que sucede en el país y realizar comparativos de lo que sucede en diversas zonas de México.

\section{Referencias bibliográficas}

Agulló-Tomás, E. (1998). La centralidad del trabajo en el proceso de construcción de la identidad de los jóvenes: una aproximación psicosocial. Psicothema, 10(1), 153-165.

Agulló-Tomás, E., Llosa, J. A., \& Agulló-Tomás, M. S. (2018). Trabajo indecente, contexto actual e implicaciones. En J. L ÁlvaroEstramiana (Ed.), La Interacción Social (Homenaje a José Ramón Torregrosa). Madrid, España: CIS Academia.

Bauman,Z.(1999). Trabajo, consumismo y nuevos pobres. Barcelona, España: Gedisa.

Blanco, A. (1997). Factores psicosociales de la vida adulta. En M. Carretero, J. Palacios, \& Á. Marchesi, Psicología evolutiva. Adolescencia, madurez y senectud (Vol. 3). Madrid, España: Alianza Editorial.

Canivet, C., Aronsson, G., Bernhard-Oettel, C., Leineweber, C., Moghaddassi, M., Stengård, J., Westerlund, H. \& Östergren, P. (2017). The negative effects on mental health of being in a non-desired occupation in an increasingly precarious labour market. SSM - Population Health 3, 516-524. DOI: https://doi.org/10.1016/j. ssmph.2017.05.009

Castells, M. (2000). Globalización, sociedad y política en la era de la Información. Bitácora Urbano Territorial, 1(4), 42-53. Recuperado de https://revistas. unal.edu.co/index.php/bitacora/article/ view/18812/19705

Consejo Nacional para la Evaluación de la Política de Desarrollo Social. (2018). Pobreza y género en México: Hacia un sistema de indicadores 2010-2016. Recuperado de https://www.coneval.org.mx/Medicion/ $\mathrm{MP} / \mathrm{Paginas} / \mathrm{Pobreza}-\mathrm{y}-\mathrm{genero}-\mathrm{en}$ Mexico-2010-2016.aspx

Díaz-Carrión, I. A. (2013). Mujeres y mercado de trabajo del turismo alternativo en Veracruz. Economía, Sociedad y Territorio, 13(42), 351-380.

Gobillon, L., Meurs, D., \& Roux, S. (2015). Estimating gender differences in access to Jobs. Journal of Labor Economics, 33, (2), 317, 363 .

Gutiérrez, T. (2016). El auge del empleo precario en Europa. Conceptos, indicadores y efectos de la crisis económica mundial. Revista Internacional del Trabajo, 135 (4), 515, 548.

Instituto Nacional de Estadística Geografía e Investigación. (2016). Estadísticas a propósito del...día de la madre (10 de mayo), datos nacionales. México: Instituto Nacional de Estadística Geografía e Investigación. Recuperado de http://www.inegi.org.mx/saladeprensa/ aproposito/2016/madre2016 0.pdf

Lara-Aldave, S., \& Vizcarra-Bordi, I. (2008). Políticas ambientales-forestales y capital 
social femenino mazahua. Economía, Sociedad y Territorio, VIII (26), 477-515.

Martínez, B. (2003). Género, sustentabilidad y empoderamiento en proyectos ecoturísticos de mujeres indígenas. Revista de Estudios de Género. La ventana, (17), 188-217.

Mendoza, M. M., \& Chapulín, J. de V. (2015). Turismo, trabajo femenino y empoderamiento de las mujeres en bahías de Huatulco, Oaxaca - México. Estudios y perspectivas en turismo, 24(2), 316-335.

Moscone, F., Tosetti, E., \& Vittadini, G. (2016). The impact of precarious employment on mental health: The case of Italy. Social Science \& Medicine, 158, 86-95.

Neffa, J. C. (2010). La transición desde los "verdaderos empleos" al trabajo precario. En E. De la Garza \& J. C. Neffa, Trabajo, identidad y acción colectiva (pp. 43-80). México: Universidad Autónoma Metropolitana. Recuperado de http://sgpwe.izt.uam.mx/pages/egt/ publicaciones/libros/trabajoidentidad/ Medellin.pdf

Organización Internacional del Trabajo. (2019). Informe Mundial sobre Salarios 2018/2019. Qué hay detrás de la brecha salarial de género. Ginebra: Organización Internacional del Trabajo. Recuperado de https://www.ilo.org/wcmsp5/groups/ public/---dgreports/---dcomm/---publ/ documents/publication/wcms 650653.pdf
Organización Internacional del Trabajo. (2012). Del trabajo precario al trabajo decente. Ginebra: Organización Internacional del Trabajo.

Organización para la Cooperación y el Desarrollo Económicos. (2017a). Brecha de género en la tasa de empleo, 2016. OCDE. Recuperado de https://www.oecd.org/ mexico/Gender2017-MEX-es.pdf

Organización para la Cooperación y el Desarrollo Económicos. (2017b). Los avances en materia de igualdad de género son demasiado lentos, dice OCDE. OCDE. Recuperado de https://www.oecd.org/ centrodemexico/medios/los-avancesen-materia-de-igualdad-de-genero-sondemasiado-lentos-dice-ocde.htm

Pienaar, J., De Witte, H., Hellgren, J., \& Sverke, M. (2013). The cognitive/affective distinction of job insecurity: Validation and differential relations. Southern African Business Review, 17(2), 1-22.

Soares, D., Castoreña, L., \& Ruiz, E. (2005). Mujeres y hombres que aran en el mar y en el desierto: Reserva de la Biosfera El Vizcaíno, BCS. Revista Frontera Norte, 17(34), 67-102.

Vargas, E. (2010). Desarrollo, trabajo y empoderamiento en el contexto del turismo (Puerto Vallarta), desde un enfoque de género (Tesis doctoral). México.

Young, M. (2010). Gender Differences in Precarious Work Settings. Relations industrielles, 65(1), 74-97. 